\title{
Continuously Happened Three Mechanical Complications after Acute Myocardial Infarction: Report of a Rare Case
}

\author{
Hideki Tsubota*, Genichi Sakaguchi, Yoshio Arai, Akira Marui \\ Department of Cardiovascular Surgery, Kokura Memorial Hospital, Kitakyushu, Japan \\ Email: *tsubota07@gmail.com
}

How to cite this paper: Tsubota, H., Sakaguchi, G., Arai, Y. and Marui, A. (2018) Continuously Happened Three Mechanical Complications after Acute Myocardial Infarction: Report of a Rare Case. World Journal of Cardiovascular Surgery, 8, 151-156. https://doi.org/10.4236/wjcs.2018.89014

Received: August 6, 2018

Accepted: September 9, 2018

Published: September 12, 2018

Copyright (๑) 2018 by authors and Scientific Research Publishing Inc. This work is licensed under the Creative Commons Attribution International License (CC BY 4.0).

http://creativecommons.org/licenses/by/4.0/

\begin{abstract}
Revascularization to infarcted area after left ventricular free-wall rupture has been controversial. A 68-year-old man with acute myocardial infarction presented to our hospital and developed a left ventricular free-wall rupture. We repaired the left ventricular oozing rupture without culprit artery revascularization, however, followed by papillary muscle rupture and left ventricular blow-out rupture, which resulted in sudden death.
\end{abstract}

\section{Keywords}

Acute Myocardial Infarction, Left Ventricular Free-Wall Rupture, Papillary Muscle Rupture

\section{Introduction}

Left ventricular free wall rupture is a well-recognized complication of myocardial infarction. Surgical repair is the only life-treating option for this critical condition, however, culprit artery revascularization after left ventricular free-wall rupture has been controversial. Concomitant revascularization at the time of repair may prevent the possible occurrence of postoperative ischemia [1], however, there are no conclusive data to support the revascularization. We report a rare case of surgical repair of left ventricular oozing rupture without culprit artery revascularization followed by papillary muscle rupture and left ventricular blow-out rupture. This case may suggest the significance of culprit artery revascularization of left ventricular free-wall rupture to prevent additional mechanical complications.

\section{Case Report}

A 68-year-old man presented at our emergency department with chest pain. 
Upon admission, the serum level of the MB fraction of creatinine kinase was 2.4 $\mathrm{ng} / \mathrm{mL}$ and electrocardiographic findings were normal. Echocardiography revealed normal left ventricular wall motion without a thin or echo-dense wall or pericardial effusion. Unstable angina was diagnosed and elective coronary angiography was scheduled. However, the chest pain worsened and his systolic blood pressure dropped to $60 \mathrm{mmHg} 10$ hours later. Electrocardiography showed an ST-segment elevation in the inferior and lateral leads, and serum creatine kinase and troponin I levels were elevated. Emergency coronary angiography revealed an occluded circumflex coronary artery without collateral flow and severe stenosis at the left anterior descending artery and diagonal branch (Figure 1). The right coronary artery was intact. Echocardiography indicated massive pericardial effusion with cardiac tamponade. Fresh bloodyfluid was drained by pericardiocentesis. Acute left ventricular free-wall rupture requiring emergency surgery was suspected, but catheter intervention was not indicated.

The heart was exposed through a median sternotomy. Slight bloody oozing was found at the lateral wall of the left ventricle near the apex, but a myocardial tear was not obvious. An oozing left ventricular free-wall rupture was diagnosed. The rupture site was repaired with a TachoSil ${ }^{\circledR}$ fibrin sealant patch (Nycomed, Linz, Austria) without sutures or cardiopulmonary bypass support. Thereafter, revascularization proceeded via off-pump anastomosis of the left internal thoracic artery (LITA) to the left anterior descending artery (LAD) and the saphenous vein graft (SVG) to the diagonal branch. Bypass to the circumflex artery was not performed because it was considered to encourage the bleeding at the ruptured site.

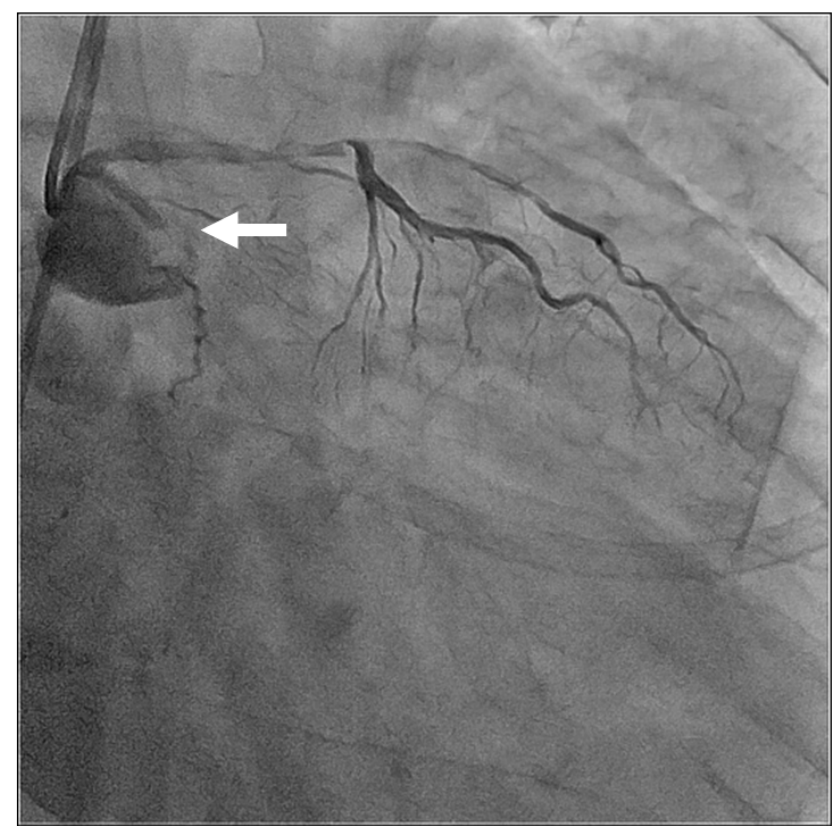

Figure 1. Emergency coronary angiography findings. Circumflex coronary arteryis occluded (arrow) and the left anterior descending artery and diagonal branch are severely stenosed. 
The patient was extubated on POD4, but respiratory failure that occurred on POD 20 required re-intubation. A chest X-ray indicated severe pulmonary edema and moderate pleural effusion. Transthoracic echocardiography displayed an ejection fraction of $60 \%$ without regional wall motion abnormalities. We treated the heart failure with diuretics and catecholamines, which did little to improve his status. Transesophageal echocardiography on POD 24 showed severe mitral regurgitation with partial papillary muscle rupture (Figure 2). Emergency mitral valve replacement proceeded on POD25. The anterolateral papillary muscle was partially transected and anterior mitral leaflet was prolapsed. The St Jude Medical Epic valve (St Jude Medical, Inc, St Paul, Minn) was implanted after resected the anterolateral papillary muscle and anterior mitral leaflet, the posterior mitral leaflet was preserved.

The postoperative course was uneventful for one week. The patient then fell into cardiogenic shock followed by cardiorespiratory arrest on POD32 and was resuscitated via immediate pericardiocentesis, but then died. Autopsy findings were consistent with myocardial infarction in the lateral wall and a left ventricular free-wall rupture close to the ruptured papillary muscle (Figure 3 ). The first repaired rupture located at a different site from the second ruptured site did not have an obvious tear.

\section{Discussion}

Left ventricular free-wall rupture is one of the most devastating complications of acute myocardial infarction, occurring at a rate of around 1\% [2]. Left ventricular free-wall rupture comprises oozing and blow-out types. The oozing type,

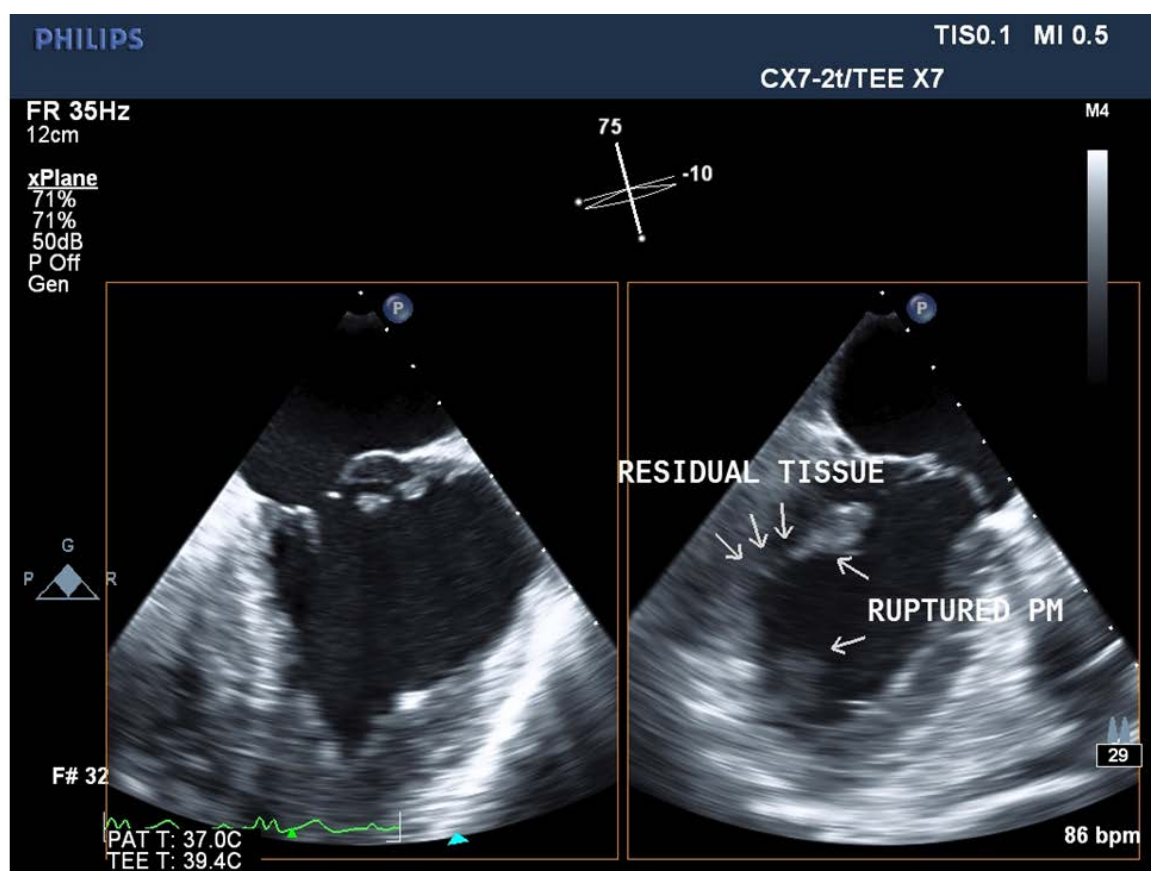

Figure 2. Transesophageal echocardiography findings. Partial papillary muscle rupture is evident. 


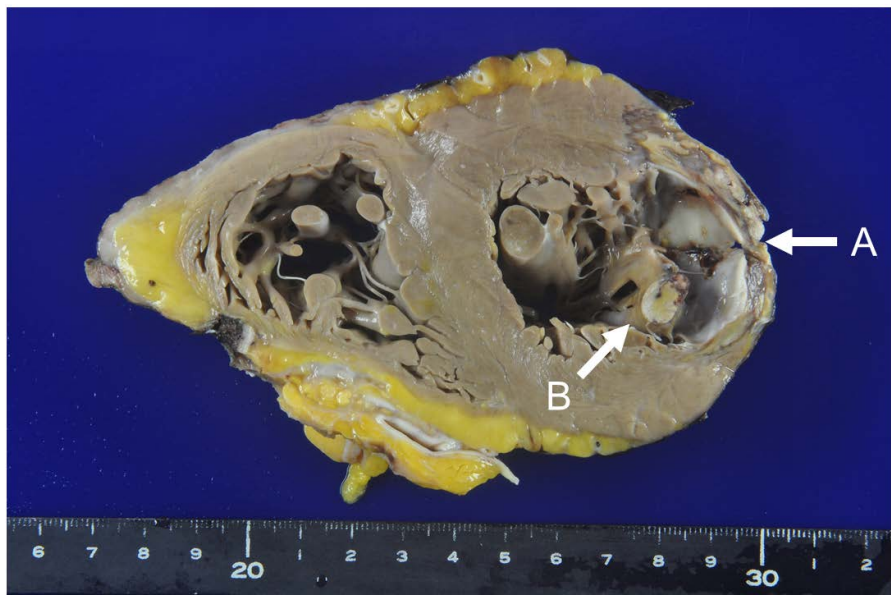

Figure 3. Pathological autopsy findings. A: Ruptured site of myocardial infarction. B: Ruptured papillary muscle.

characterized by a small perforation, allows sufficient time for diagnosis and surgery but the blow-out type is characterized by massive hemorrhage that leads to rapid circulatory disturbance and death within minutes. Our patient experienced both types of left ventricular rupture within one month.

Sutureless patch repair is effective for post-infarction left ventricular rupture [3] [4]. It is sutureless, simple, reproducible, and a cardiopulmonary bypass that requires systemic heparinization can be avoided, which confers an obvious advantage for hemostasis. Sakaguchi et al. summarized a cohort study of 32 consecutive patients [3] in which left ventricular ruptures were repaired using autologous pericardial patches and glue with an operative mortality of $15.6 \%$, which was deemed satisfactory. The operative mortality was $23.5 \%$ in a cohort study of 17 consecutive patients with post-infarction left ventricular ruptures that were repaired using a Gore-Tex ${ }^{\circledR}$ patch and glue without a cardiopulmonary bypass [4].

$\mathrm{TachoSil}^{\circledR}$ is a fibrin tissue adhesive that has been advocated to prevent further rupture of oozing ruptures. Muto et al. reported good outcomes of treating oozing left ventricular ruptures with $\mathrm{TachoSil}^{\circledR}$ in 2005 [5]. Raffa et al. described six consecutive patients who were treated with $\mathrm{TachoSil}^{\circledR}$ patches and decided that the application of these patches to a beating heart is their first choice to achieve hemostasis in left ventricular rupture [6]. The first oozing rupture in our patient was cured using a TachoSil ${ }^{\circledR}$ patch. However, the subsequent blow-out rupture occurred one month later in an area that was not covered by the patch. We should have patched a more extensive area of potential necrotic change.

Mitral valve regurgitation due to papillary muscle rupture occurs in $1 \%$ to $3 \%$ of patients after acute myocardial infarction [7] [8] [9]. Although rare, papillary muscle rupture is a catastrophic complication with a poor outcome in the absence of emergency surgical repair. Most papillary muscle ruptures occur within one week after myocardial infarction, but rupture can occur several weeks after myocardial infarction. The papillary muscle ruptured at over two weeks after 
myocardial infarction in our patient. In fact, we did not assume papillary muscle rupture and transthoracic echocardiography did not detect any papillary muscle abnormalities, but transesophageal echocardiography revealed detailed mitral valve pathology.

The necessity of concomitant myocardial revascularization is controversial. Solis et al. described that concomitant revascularization does not improve the outcomes [10]. On the other hand, Reardon et al. advocated the revascularization at the time of repair to prevent the possible occurrence of postoperative ischemia, however, they concluded that there are no conclusive data to support the revascularization because the number of reported survivors is small, and there is no substantial long-term follow-up of bypassed and non bypassed patients [11]. Leva et al. reported an excellent early and long-term outcome with complete myocardial revascularization in the non-infarcted area [1]. In present case, we performed the revascularization to LAD and diagonal branch, but not to circumflex branch which was the culprit artery.

Our patient developed three rare, serious complications of acute myocardial infarction in succession. The present findings suggest that the entire affected area should be reinforced to avoid potential necrotic change, and patients who present with heart failure of unknown cause should be immediately assessed by transesophageal echocardiography, and the revascularization to the infarcted area may prevent the sequential ischemic events.

\section{Conflicts of Interest}

The authors declare no conflicts of interest regarding the publication of this paper.

\section{References}

[1] Leva, C., Bruno, P.G., Gallorini, C., Lazzarini, I., Musazzi, G., Vittonati, L., et al. (2006) Complete Myocardial Revascularization and Sutureless Technique for Left Ventricular Free Wall Rupture: Clinical and Echocardiographic Results. Interactive Cardio Vascular and Thoracic Surgery, 5, 408-412.

https://doi.org/10.1510/icvts.2006.131037

[2] Patel, M.R., Meine, T.J., Lindblad, L., Griffin, J., Granger, C.B., Becker, R.C., et al. (2006) Cardiac Tamponade in the Fibrinolytic Era: Analysis of $>100000$ Patients with ST-Segment Elevation Myocardial Infarction. American Heart Journal, 151, 316-322. https://doi.org/10.1016/j.ahj.2005.04.014

[3] Sakaguchi, G., Komiya, T., Tamura, N. and Kobayashi, T. (2008) Surgical Treatment for Postinfarction Left Ventricular Free Wall Rupture. The Annals of Thoracic Surgery, 85, 1344-1346. https://doi.org/10.1016/j.athoracsur.2007.12.073

[4] Canovas, S.J., Lim, E., Dalmau, M.J., Bueno, M., Buendía, J., Hornero, F., et al. (2003) Midterm Clinical and Echocardiographic Results with Patch Glue Repair of Left Ventricular Free Wall Rupture. Circulation, 108, II-237-40. https://doi.org/10.1161/01.cir.0000089042.80722.7a

[5] Muto, A., Nishibe, T., Kondo, Y., Sato, M., Yamashita, M. and Ando, M. (2005) Sutureless Repair with TachoComb Sheets for Oozing Type Postinfarction Cardiac 
Rupture. The Annals of Thoracic Surgery, 79, 2143-2145.

https://doi.org/10.1016/j.athoracsur.2003.12.033

[6] Raffa, G.M., Tarelli, G., Patrini, D. and Settepani, F. (2013) Sutureless Repair for Postinfarction Cardiac Rupture: A Simple Approach with a Tissue-Adhering Patch. The Journal of Thoracic and Cardiovascular Surgery, 145, 598-599. https://doi.org/10.1016/j.jtcvs.2012.08.049

[7] Tcheng, J.E., Jackman Jr., J.D., Nelson, C.L., et al. (1992) Outcome of Patients Sustaining Acute Ischemic Mitral Regurgitation during Myocardial Infarction. Annals of Internal Medicine, 117, 18-24. https://doi.org/10.7326/0003-4819-117-1-18

[8] Thompson, C.R., Buller, C.E., Sleeper, L.A., Antonelli, T.A., Webb, J.G., Jaber, W.A., et al. (2000) Cardiogenic Shock due to Acute Severe Mitral Regurgitation Complicating Acute Myocardial Infarction: A Report from the SHOCK Trial Registry. Should We Use Emergently Revascularize Occluded Coronaries in Cardiogenic shock? Journal of the American College of Cardiology, 36, 1104-1109.

https://doi.org/10.1016/S0735-1097(00)00846-9

[9] French, J.K., Hellkamp, A.S., Armstrong, P.W., Cohen, E., Kleiman, N.S., O'Connor, C.M., et al. (2010) Mechanical Complications after Percutaneous Coronary Artery Intervention in ST-Elevation Myocardial Infarction (from APEX-AMI). American Journal of Cardiology 105, 59-63. https://doi.org/10.1016/j.amjcard.2009.08.653

[10] Solis, C., Pujol, D. and Mauro, V. (2009) Left Ventricular Free Wall Rupture after Acute Myocardial Infarction. Revista Argentina de Cardiología, 77, 395-404.

[11] Reardon, M.J., Carr, C.L., Diamond, A., Letsou, G.V., Safi, H.J., Espada, R., et al. (1997) Ischemic Left Ventricular Free Wall Rupture: Prediction, Diagnosis, and Treatment. The Annals of Thoracic Surgery, 64, 1509-1513. https://doi.org/10.1016/S0003-4975(97)00776-5 